\title{
Single-molecule sizing through nano-cavity confinement
}

Raphael P. B. Jacquat ${ }^{1,3, \dagger}$, Georg Krainer ${ }^{1, \dagger}$, Quentin Peter ${ }^{1, \dagger}$, Ali Nawaz Babar ${ }^{1}$, Oliver Vanderpoorten $^{1,2,4}$, Catherine K. Xu${ }^{1}$, Clemens F. Kaminski ${ }^{2}$, Ulrich F. Keyser ${ }^{3}$, Jeremy J. Baumberg ${ }^{3}$ and Tuomas P. J. Knowles ${ }^{1,3, *}$

${ }^{1}$ Yusuf Hamied Department of Chemistry, University of Cambridge, Lensfield Road, Cambridge, CB2 1EW, UK

${ }^{2}$ Department of Chemical Engineering and Biotechnology, University of Cambridge, Philippa Fawcett Drive, Cambridge, CB3 OAS, UK

${ }^{3}$ Cavendish Laboratory, Department of Physics, University of Cambridge, J. J. Thomson Avenue, Cambridge, CB30HE, UK

${ }^{4}$ Department of Physics and Technology, UiT The Arctic University of Norway, Troms $\emptyset$, Norway

$\dagger$ These authors contributed equally to this work

*To whom correspondence should be addressed: tpjk2@cam.ac.uk 


\begin{abstract}
An approach relying on nano-cavity confinement is developed in this paper for the sizing of nanoscale particles and single biomolecules in solution. The approach, termed nano-cavity diffusional sizing (NDS), measures particle residence times within fluidic nano-cavities to determine their hydrodynamic radii. Using theoretical modelling and simulation, we show that the residence time of particles within nano-cavities above a critical timescale depends on the diffusion coefficient of the particle, which allows estimation of the particle's size. We demonstrate this approach experimentally through measurement of particle residence times within nano-fluidic cavities using single-molecule confocal microscopy. Our data show that the residence times scale linearly with the sizes of nanoscale colloids, protein aggregates and single DNA oligonucleotides. NDS thus constitutes a new single molecule optofluidic approach that allows rapid and quantitative sizing of nanoscale objects for potential application in nanobiotechnology, biophysics, and clinical diagnostics.
\end{abstract}




\section{Introduction}

Many important biomolecules, including proteins and protein assemblies as well as natural and synthetic biopolymers and colloids have sizes in the nanometre range. ${ }^{1,2}$ Achieving rapid, accurate, and reliable measurement of their sizes under native solution conditions has therefore become a key objective in many areas of current research including nanobiotechnology, biophysics, and clinical diagnostics. ${ }^{3-5}$ For example, sizing of proteinaceous particles at nanometre scales is critical in studies that further our understanding of protein misfolding and aggregation processes, which lie at the heart of a wide range of human diseases. ${ }^{6-8}$ Moreover, characterising the assembly state of biomacromolecules is important when assessing, for example, biopharmaceutical product stability and efficacy of proteins or biocolloids in drug delivery systems and formulations. ${ }^{9-11}$ Sizing techniques are therefore considered work horses in many areas of fundamental and applied science. ${ }^{3,12}$ Hence, the development of experimental approaches for high sensitivity detection and characterization of nanoscale entities in the fluid phase remains an area of great current interest.

Several techniques are available in order to measure the nanoscale size of proteins and nanocolloids in solution. ${ }^{3-5}$ Most of them are based on determining the particle's diffusion coefficient $D$ in solution, which is related via the Stokes-Einstein equation to the hydrodynamic radius $R_{\mathrm{H}}$ of the particle. One of the most widely used techniques is dynamic light scattering (DLS). ${ }^{13}$ Other widely used methods include nuclear magnetic resonance (NMR)-based techniques (e.g., pulse-field gradient NMR), ${ }^{14}$ chromatographic techniques, ${ }^{15}$ and surface deposition microscopy, like atomic force microscopy (AFM) or scanning/transmission electron microscopy (SEM/TEM). ${ }^{16,17}$ These techniques suffer from relatively high sample consumption and long acquisitions times or surface immobilization, and often require sophisticated instrumentation. In recent years, a number of techniques have been established that operate with minimal sample requirements and sensitivities down to the single molecule regime, and operate directly in solution. These include microfluidic techniques ${ }^{18,19}$ such as microfluidic diffusional sizing (MDS) ${ }^{8}$ Taylor dispersion analysis (TDA), ${ }^{20}$ nanoparticle tracking analysis (NTA) ${ }^{21}$ and single molecule techniques such as fluorescence correlation spectroscopy (FCS) ${ }^{22}$ or a combination of interferometric scattering (iSCAT) microscopy ${ }^{23}$ with electrostatic trapping. ${ }^{24}$ Such fluidic and single molecule-based approaches offer great potential for the sizing of nanoparticles and nano-colloids, however, they are often limited in the size range that can be detected and require complex models to analyse the size distributions.

In this work, we introduce a nanofluidic approach, termed nanocavity diffusional sizing (NDS), which allows for the robust determination of the sizes of single particles directly in solution through measurement of particle residence times within fluidic nano-cavities. The approach extracts sizes of nanoparticles through nano-confinement and allows sizing of particles in the range from a few nanometres up to hundreds of nanometres. The implementation and experimental realization of the NDS approach is outlined in Figure 1. The observation volume of a confocal microscope is placed within one of the trapping cavities of a nanofluidic device, which itself is filled with an aqueous solution that contains the biomolecule-of-interest (Figure 1a). The chip design is shown in Figure 1b. To extract the sizes of single molecules, time trajectories of particles diffusing into and out of the observation volume are recorded, and residence time distributions are extracted (Figure 1c). Fitting of the obtained residence time histograms with a quantitative model provides $R_{\mathrm{H}}$ and, thus, the size of the nanoparticle-ofinterest (Figure 1c). 
a

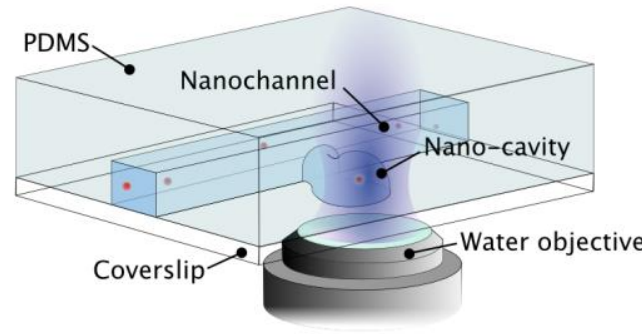

b
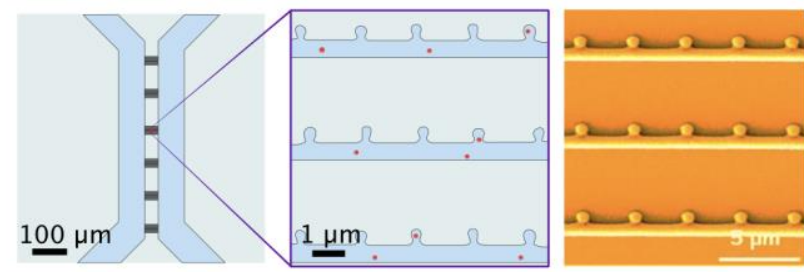

C
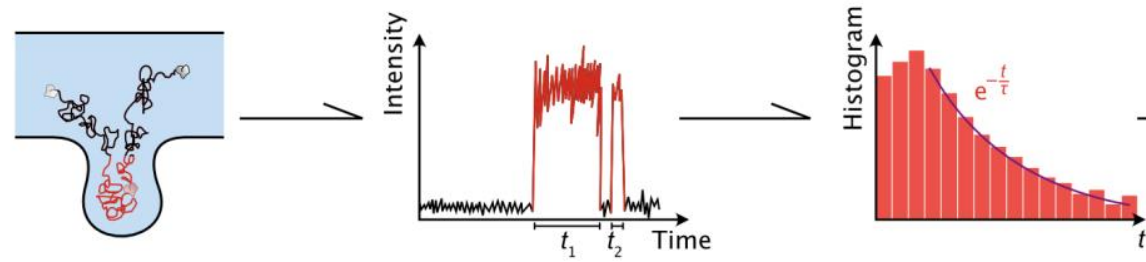

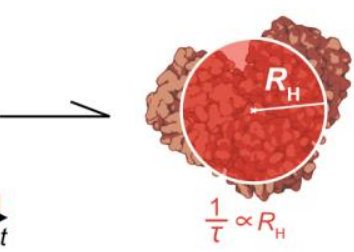

Figure 1. Principle of nanocavity diffusional sizing (NDS). (a) Three-dimensional illustration of the experimental implementation of NDS showing the positioning of the confocal detection volume inside the nanocavity of the nanofluidic chip. A high numerical aperture objective is used for single molecule detection of molecules within the nano-cavity of the chip, which is fabricated by hybrid lithography and moulding in PDMS. Biomolecules are depicted in red. (b) Schematic of the nanofluidic chip used for NDS measurements. The nanocavities are located adjacent to nanofluidic channels on a microfluidic chip. A SEM image of the chip is depicted in the right panel. (c) Workflow of the sizing experiment. First, the particles are detected by confocal microscopy as they diffuse into and out of the nano-cavity. Then, the residence times $t$ are extracted from the recorded time trace and binned in a residence time histogram according to the occurrence of residence time. This histogram is then fit with an exponential function of the type $e^{-\frac{t}{\tau}}$, from which the hydrodynamic radius $R_{\mathrm{H}}$ can be extracted. The coefficient $\tau$ is inversely proportional to the hydrodynamic radius.

\section{Theory and Simulation}

We first developed an analytical model to examine the diffusive behaviour of particles within nano-cavities and to quantitatively describe how particle size relates to residence time. This allowed us to assess the scaling behaviour of particle residence times and provided us with a theoretical framework for the analysis of our experimental results. We modelled the diffusion of particles as Brownian motion with reflective boundary conditions on the walls. No other potential was considered as we work under conditions where the direct electrostatic interactions between analyte particle and the cavity walls are negligeable as the particle is typically separated by more than the screening length from the walls. The nano-cavity, as shown in Figure 1a, was modelled as a cavity of rectangular shape (Figure 2a), which was perpendicularly connected to adjacent nanochannels. The particle, in the model, can therefore only enter and exit the cavity by diffusing perpendicular to the nanochannel axis. Assuming that diffusion is isotropic, the model, for the case of a rectangular cavity, can therefore be reduced to a one-dimensional (1D) diffusion problem. 
a
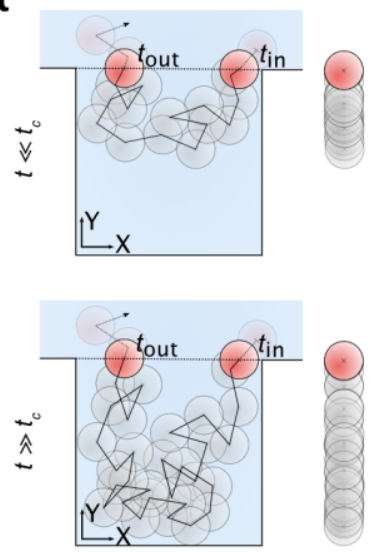

C

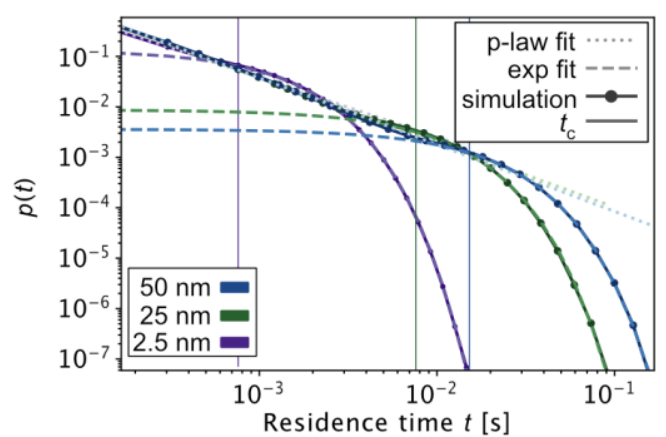

b
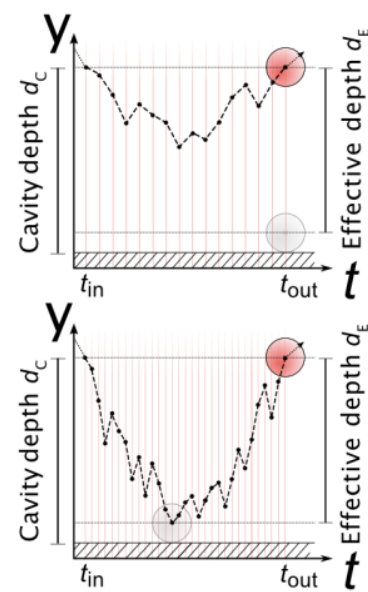
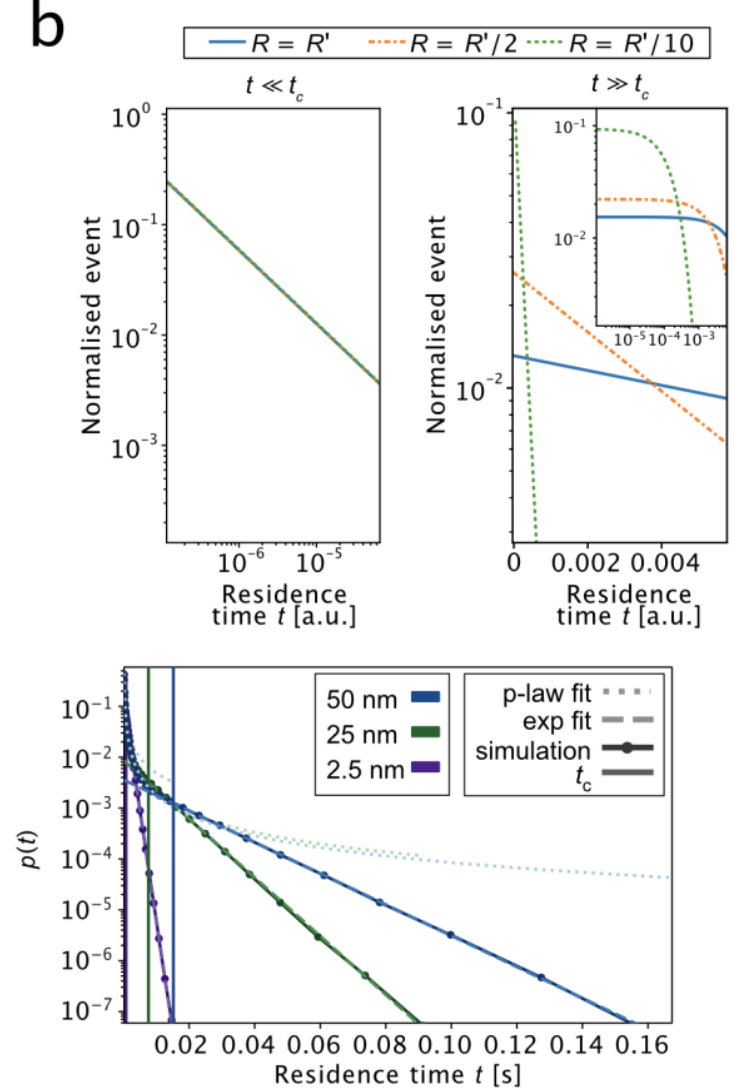

Figure 2. Theory and simulation of diffusion under nano-confinement. (a) For a particle diffusing within a nano-cavity, two diffusive scenarios can be distinguished: (i) the particle enters the cavity and exits it without reaching the bottom wall (top panels) or (ii) the particle enters the cavity and reaches the bottom of the well before exiting it (bottom panels). The right panels show displacement in time along the y-axis of the diffusion processes. The particle enters at time $t_{\text {in }}$ and exits at time $t_{\text {out }}$. The depth of the cavity is $d_{\mathrm{c}}$, and $d_{\mathrm{E}}$ denotes the effective cavity depth $\left(d_{\mathrm{E}}=d_{\mathrm{c}}-R\right)$, with $R$ being the radius of the particle. (b) Analytical modelling of particle diffusion at short timescales $\left(t<<t_{\mathrm{c}}\right.$, left panel) and long timescales $\left(t \gg t_{\mathrm{c}}\right.$, right panel). $t_{\mathrm{c}}$ denotes the critical time that separates the two regimes and corresponds to the mean time for a particle with diffusion coefficient $D$ to reach the end of the nano-trap (see main text). Shown are residence time probability plots. At short timescales $\left(t<<t_{\mathrm{c}}\right)$, the residence time is independent of the size of the particle. At long timescales $\left(t>t_{\mathrm{c}}\right)$, the residence time follows an exponential decay which is dependent on the size of the particle. Modelled were three particles with different radii at $R^{\prime}$ fixed: $R=R^{\prime}$ (blue), $R=R^{\prime} / 2$ (orange), and $R=R^{\prime} / 10$ (green). Diffusion was modelled as a 1D random walk. (c) Simulation results for the diffusion of particles within a nano-cavity. Shown are residence time probability plots (log-log plot, left panel; linear-log plot, right panel) for particles of different seizes ( $50 \mathrm{~nm}$, blue; $25 \mathrm{~nm}$, green; $2.5 \mathrm{~nm}$, purple). At short residence time, particles are scale invariant, as evident by a linear behaviour in the log-log plot (i.e., power law behaviour). At long timescales, particle residence times exhibit an exponential decay, which is dependent on the size of the particle, due to the linear behaviour in the linear-log plot. Data points represent simulation results. Long and short dashed lines depict fits of the simulation data by power law and exponential functions, respectively.

The residence time of a particle is determined by the probability of the particle exiting the cavity over a given period-of-time. Representative time trajectories of a particle entering and exiting the cavity are shown in Figure 2a. The particle enters the cavity at time $t=0$ and the time points to describe particle entry and exit are denoted as $t_{\text {in }}$ and $t_{\text {out }}$, which yields the residence time $t=t_{\text {out }}-t_{\text {in. }}$. The length of the cavity is $d_{E}$, where $d_{E}$ is the effective depth of the cavity and is given by the depth of the cavity $d_{C}$ minus the particle radius $R$ :

$$
d_{E}=d_{C}-R
$$


As depicted in Figure 2a,b, two regimes can be observed in the residence time distribution: a short and a long timescale regime. These two regimes are separated by a critical time $t_{\mathrm{c}}$, which corresponds to the mean time for a particle with diffusion coefficient $D$ to reach the bottom wall of the nano-cavity according to:

$$
t_{c}=d_{E}^{2} / 2 D
$$

For short timescales, the particle resides within the cavity only for a short period of time such that it typically does not diffuse to the far end of the cavity (Figure 2a, upper panels). The probability distribution of the particle position inside the cavity is therefore concentrated near the original position. The distribution in this regime can be described by an unconstrained random walk model and is given by the first passage time density:

$$
p(t)=\frac{\left|y-y_{0}\right|}{\sqrt{4 \pi D t^{3}}} \exp \left(-\frac{\left(y-y_{0}\right)^{2}}{4 D t}\right)
$$

where $y$ is the coordinate position between the inside/outside of the cavity (position where $t=0)$ and $y_{0}$ is the distance that the particle diffuses inside the cavity in y-direction. For very small $\Delta y$, such that $\frac{\Delta y^{2}}{4 D} \ll t \ll t_{c}$, the density can be expressed as:

$$
p(t)=\frac{\Delta y}{\sqrt{4 \pi D t^{3}}} \propto t^{-\frac{3}{2}}
$$

Accordingly, for the system at $\frac{\Delta y^{2}}{4 D} \ll t \ll t_{c}$, the residence time follows a power law, which is independent of $D$, because $\Delta y$ scales with $\sqrt{D}$ (see Supporting Information). This behaviour is displayed in Figure 2b, left panel for particles with different diffusion coefficients.

For long timescales, the cavity starts playing a role in the diffusion process, as the particle has enough time to explore the confined volume through diffusion. The free random walk model can therefore no longer be applied. As described in the Supporting Information and detailed in an analogous situation in Ref. ${ }^{25}$ (Equation 5.47 therein), the residence time distribution in this regime has an exponential dependence:

with the decay time being:

$$
p(t) \propto e^{-\frac{t}{\tau}}
$$

$$
\tau=\frac{4 d_{E}^{2}}{D \pi^{2}}
$$

Accordingly, $\tau$ is inversely proportional to $D$. Hence, for the system at $t \gg t_{c}$ the size of the particle is linked to the decay time. This scaling behaviour is shown in Figure 2b, right panel for particles with different diffusion coefficients. Residence time measurements of particles at large timescales $\left(t \gg t_{c}\right)$ allow estimations of the size of particles. This model can therefore size particles without the requirement to have an energetic contribution from any kind of trapping free energy potential.

To corroborate our results from analytical modelling, we further performed numerical simulations of a particle $1 \mathrm{D}$ random walk within a nano-cavity to extract residence time 
probability distributions for differently sized particles. The simulations were performed using a reflective boundary condition for the wall on the bottom of the cavity. A Gaussian random number generator was used to simulate diffusive steps. Details of the simulations are given in the Methods section. Obtained residence time probability distributions are shown in Figure 2c. The results are consistent with the theory above, in that, at short timescales, the particle's residence times follow a power law behaviour $\left(t^{-\frac{3}{2}}\right)$, as evident in a linear decay in the log-log plot, whereas at long timescales, the residence time decays exponentially $\left(e^{-\frac{t}{\tau}}\right)$, as evident in the linear decay in the linear-log plot. Fitting of the simulation results recovered the initial input values with a relative error of $0.5 \%$, demonstrating the robustness of our analysis approach.

\section{Experimental demonstration of the NDS approach}

After having explored the possibility of sizing particles in nano-cavities on a theoretical basis, we next set out to demonstrate the NDS approach experimentally. Conceptually, the experimental implementation of the NDS approach involves the following steps: First, the durations of trapping events within nano-cavities need to be recorded, from which probability distributions of residence times $p(t)$ are generated. Then, by fitting this distribution with an exponential function, Eqs. 5 and 6 can be used to compute the diffusion coefficient from the decay time of this exponential.

Based on these considerations, we set out to experimentally implement the NDS approach for the sizing of single particles in solution. We made use of a nano-fluidic device, previously developed in our laboratory, to measure particle residence times within nano-cavities. ${ }^{26,27} \mathrm{~A}$ schematic of the fluidic platform is shown in Figure 3a, upper panel. The device consists of arrays of nano-cavities which are connected to nanofluidic channels. These nanofluidic functionalities lie in between two microfluidic reservoirs with inlets and outlets that serve as fill ports for the sample solution. The nano-cavities are of cylindrical shape and have a radius of $350 \mathrm{~nm}$ and a height of $650 \mathrm{~nm}$. The connecting nano-fluidic channels are $650 \mathrm{~nm}$ wide and $750 \mathrm{~nm}$ high. SEM images of the channel and nanocavity geometries are shown in Figure 3a, lower panels. The fabrication of the polydimethylsiloxane (PDMS)-silica devices by UV and 2-photon hybrid lithography (2PL) is detailed in the Methods section. For the detection of single particles, we made use of confocal fluorescence spectroscopy. Samples were excited with a continuous wave diode laser and their fluorescence collected using avalanche photodiodes, which allowed us to readout the fluorescent signal of molecules with high sensitivity and monitor their residence times within nano-cavities with high temporal resolution. A schematic of the confocal microscope equipped with a motorized stage for precise placement of the confocal volume is shown in the Supporting Information. 
a

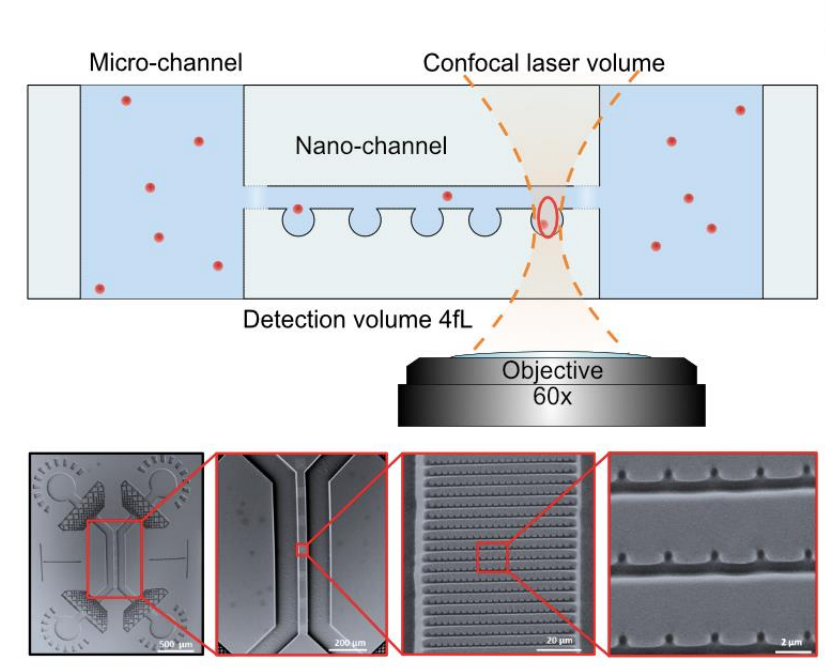

C

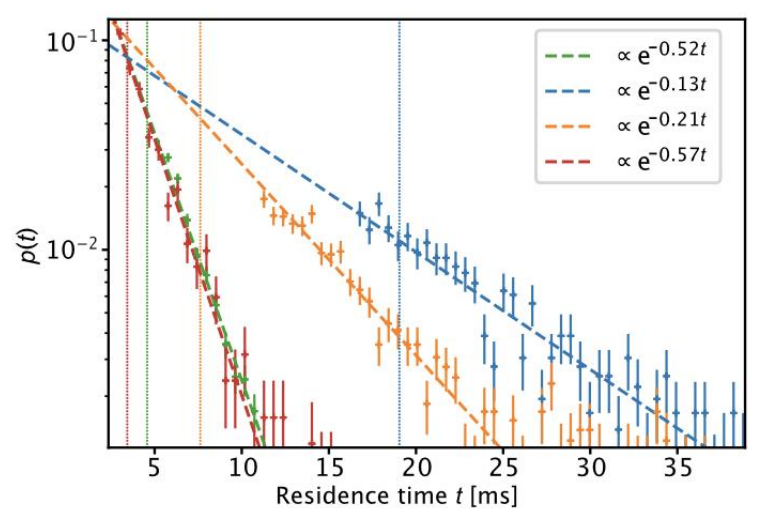

b

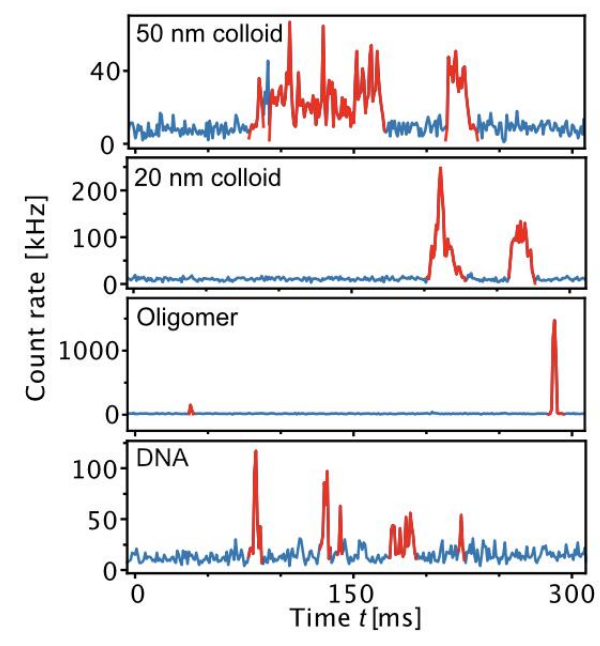

d

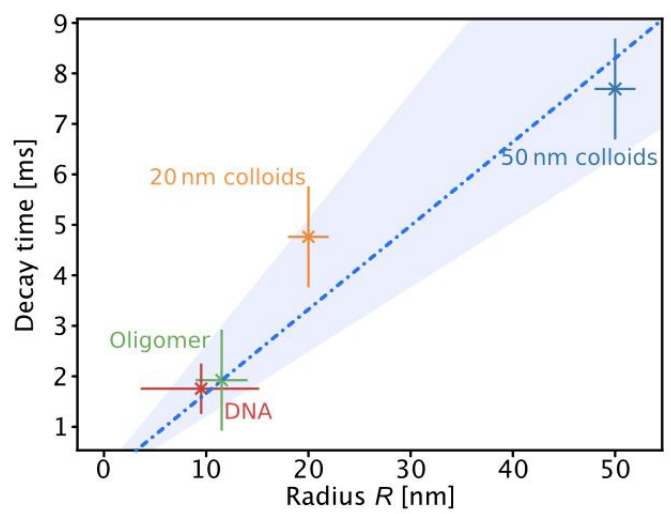

Figure 3. Nano-fluidic diffusional sizing (NDS) of single particles in solution. (a) Experimental setup of the NDS experiment. The observation volume of the confocal microscope is placed within a nano-cavity. Fluorescence of particles or biomolecules of interest are observed as they diffuse in and out of the confocal volume. Lower panel: SEM micrographs of the nanofluidic device with nano-cavity functionalities used in NDS experiments. PDMS nanofluidic device imprints were fabricated via hybrid UV mask lithography and 2-photon lithography. SEM micrographs were adapted from Vanderpoorten et al. ${ }^{26}$. (b) Exemplary time traces from fluorescence detection of nano-colloids, $\alpha$-synuclein protein oligomers, and DNA oligonucleotides. Highlighted in red are the times when a particle was present within the confocal detection volume. The bin time is $1 \mathrm{~ms}$ in all traces. (c) Normalized residence time probability histograms of particles within the nano-cavity. The data were fit with an exponential function of the form $e^{-\frac{t}{\tau}}$. The slope of the curves gives the decay time. The dotted line corresponds to the critical time $t_{c}$ for each species (d) Extracted decay times versus hydrodynamic radii. The dotted line corresponds to the fit of the data.

Using this optofluidic platform, we probed the residence times of nanoscale particles for size determination by NDS. We performed measurements on fluorescent nanoscale colloids ( 50 and $20 \mathrm{~nm}$ radius), fluorescently labelled oligomeric aggregates of the protein $\alpha$-synuclein with average sizes of around $11.5 \mathrm{~nm}$, and fluorescently labelled DNA oligonucleotides (45 bp, $9.5 \mathrm{~nm}$ ). Sample solutions were injected into the fluidic device and the confocal observation volume parked in the middle of one of the nano-cavities of the device. After a short equilibration period to ensure hydrostatic balance, single molecule fluorescence of particles diffusing into and out of the well were recorded. Exemplary time traces are shown in Figure 3b. As anticipated from our theoretical consideration, larger particles/molecules resided longer within the nano-wells as compared to smaller ones. For each detected event, we extracted the associated residence times. Individual residence times were pooled in a histogram to obtain residence time histograms. Due to the nature of the measurement, short residence 
events are under-sampled, which would create an artefact in the distribution (see Supporting Information). A threshold was therefore applied in order to represent residence times only at longer timescales. Moreover, residence times at short timescales are scale invariant; hence for size determination this regime can be omitted (see Theory and Simulations above).

The experimentally obtained residence time distributions for the four tested species are shown in Figure 3c. The residence time decays follow a linear behaviour in the linear-log plot, as expected for an exponential behaviour due to the biased random walk of the particles within the nano-cavity, as predicted from our theoretical modelling and simulations (see above). Accordingly, we fitted the data with an exponential function of the form $e^{-\frac{t}{\tau}}$. This allows extracting a decay time $\tau$, which is proportional to the size of the particle according to the theory derived above, considering also the effective well depth. A plot of the extracted decay times versus the size (i.e., $R_{\mathrm{H}}$ ) of the particles yields a linear relation (Figure 3d), as anticipated from the theory. Using such calibration, it is thus possible to size particles in solution using NDS.

Finally, we would like to note that NDS is robust against measurement noise. Single molecule experiments usually provide data at low signal-to-noise ratios. However, because size information in our approach is extracted from long timescale events, false positive events are exponentially unlikely for longer residence times, as required in our approach. In other words, the likelihood for false particle detection, which mainly happens for the detection of events on short timescales, is minimized, as NDS extracts information from long timescale events. This feature of obtaining data at the high signal-to-noise regime makes NDS robust against measurement noise and thus ensures accurate and reliable measurement of a molecule's size.

\section{Conclusions}

In this work, we have established an approach for the sizing of particle using single molecule detection and nanofluidics. The NDS approach harnesses the size-dependent diffusional escape of particles under nano-confinement to obtain size information from the particle's diffusive properties. Using our theoretical modelling and simulations, we have shown that above a critical timescale, the scaling of the particle's residence time changes from a power law, which is size independent, to an exponential, size-dependent behaviour. This realization forms the basis of our approach and yields a linear behaviour of the size of a particle versus its residence time within a nano-cavity. Using a nanofluidic chip combined with confocal spectroscopy, we have experimentally validated both the exponential scaling behaviour for nanoscale particles and biomolecules, and shown that the decay rate follows a linear behaviour with respect to the diffusion coefficient. Using such calibration, our approach can yield rapid, accurate, and reliable sizing of particles and biomolecules.

Our NDS approach lines up with other techniques such as FCS and NPT analysis in terms of measurements times, yet no correlation analysis is needed for size determination, and particle sizes from a few nanometres up to tens of nanometre can be determined, which is hardly achieved with other techniques. For example, NTA tracks particles only down to ca. $30 \mathrm{~nm}$, while FCS is most sensitive to molecules in the low nanometre regime. By nature, NDS is a single particle counting analysis technique. Such analysis offers the advantage to size heterogenous mixtures with components of different sizes and brightness. The implementation, as demonstrated here, uses fluorescence single molecule detection. However, other readout modalities including total internal reflection microscopy or scattering-based techniques (e.g., iSCAT) can be envisaged as well. 
In summary, with NDS we have presented a new single molecule optofluidic approach that allows for a rapid and quantitative sizing of nanoscale objects, opening up potential applications in areas including nanobiotechnology, biophysics, and clinical diagnostics.

\section{Associated Content}

Supporting Information. Methods, Simulation code, Mathematical model at short timescales, Mathematical model at long timescales, Considerations on concentration limit and time dependence, Under-sampling at short residence times and data selection criteria, Effect of nano-cavity non-uniformity on size determination of particles, Experimental setup used for NDS measurements

\section{Author information}

Corresponding Author.*E-mail: tpjk2@cam.ac.uk

Author Contributions. The manuscript was prepared through the contribution of all coauthors. All authors have given approval to the final version of the manuscript.

Notes. The authors declare no competing financial interest.

\section{Acknowledgements}

The work was funded by the Horizon 2020 programme through 766972-FET-OPENNANOPHLOW (T.P.J.K.). The research leading to these results has further received funding from the European Research Council under the European Union's Horizon 2020 Framework Programme through the Marie Sklodowska-Curie grant MicroSPARK (agreement $n^{\circ} 841466$; G.K.), the Herchel Smith Funds (G.K.), and the Wolfson College Junior Research Fellowship (G.K.). This work was also supported by the Engineering and Physical Sciences Research Council [grant numbers EP/L015889/1] (R.J.). The authors would also like to thank the NanoDTC for additional funding and the Maxwell Community for scientific support.

\section{References}

(1) Hamley, I. W. Protein Assemblies: Nature-Inspired and Designed Nanostructures. Biomacromolecules 2019, 20 (5), 1829-1848. https://doi.org/10.1021/ACS.BIOMAC.9B00228.

(2) Shen, Y.; Levin, A.; Kamada, A.; Toprakcioglu, Z.; Rodriguez-Garcia, M.; Xu, Y.; Knowles, T. P. J. From Protein Building Blocks to Functional Materials. ACS Nano 2021, 15 (4), 5819-5837. https://doi.org/10.1021/ACSNANO.0C08510.

(3) Modena, M. M.; Rühle, B.; Burg, T. P.; Wuttke, S. Nanoparticle Characterization: What to Measure? Adv. Mater. 2019, 31 (32), 1901556. https://doi.org/10.1002/ADMA.201901556.

(4) Mudalige, T.; Qu, H.; Van Haute, D.; Ansar, S. M.; Paredes, A.; Ingle, T. Characterization of Nanomaterials: Tools and Challenges. Nanomater. Food Appl. 2019, 313-353. https://doi.org/10.1016/B978-0-12-814130-4.00011-7.

(5) Eaton, P.; Quaresma, P.; Soares, C.; Neves, C.; de Almeida, M. P.; Pereira, E.; West, P. A Direct Comparison of Experimental Methods to Measure Dimensions of Synthetic Nanoparticles. Ultramicroscopy 2017, 182, 179-190. 
(6) Herling, T. W.; Levin, A.; Saar, K. L.; Dobson, C. M.; Knowles, T. P. J. Microfluidic Approaches for Probing Amyloid Assembly and Behaviour. Lab Chip 2018, 18 (7), 999-1016. https://doi.org/10.1039/c71c01241a.

(7) Knowles, T. P. J.; Vendruscolo, M.; Dobson, C. M. The Amyloid State and Its Association with Protein Misfolding Diseases. Nat. Rev. Mol. Cell Biol. 2014, 15 (6), 384-396. https://doi.org/10.1038/nrm3810.

(8) Arosio, P.; Müller, T.; Rajah, L.; Yates, E. V.; Aprile, F. A.; Zhang, Y.; Cohen, S. I. A.; White, D. A.; Herling, T. W.; De Genst, E. J.; Linse, S.; Vendruscolo, M.; Dobson, C. M.; Knowles, T. P. J. Microfluidic Diffusion Analysis of the Sizes and Interactions of Proteins under Native Solution Conditions. ACS Nano 2016, 10 (1), 333-341. https://doi.org/10.1021/acsnano.5b04713.

(9) Caputo, F.; Clogston, J.; Calzolai, L.; Rösslein, M.; Prina-Mello, A. Measuring Particle Size Distribution of Nanoparticle Enabled Medicinal Products, the Joint View of EUNCL and NCI-NCL. A Step by Step Approach Combining Orthogonal Measurements with Increasing Complexity. J. Control. Release 2019, 299, 31-43. https://doi.org/10.1016/J.JCONREL.2019.02.030.

(10) Shekunov, B. Y.; Chattopadhyay, P.; Tong, H. H. Y.; Chow, A. H. L. Particle Size Analysis in Pharmaceutics: Principles, Methods and Applications. Pharm. Res. 2006 242 2006, 24 (2), 203-227. https://doi.org/10.1007/S11095-006-9146-7.

(11) Kopp, M. R. G.; Pérez, A.-M. W.; Zucca, M. V.; Palmiero, U. C.; Friedrichsen, B.; Lorenzen, N.; Arosio, P. An Accelerated Surface-Mediated Stress Assay of Antibody Instability for Developability Studies. https://doi.org/10.1080/19420862.2020.1815995 2020, 12 (1). https://doi.org/10.1080/19420862.2020.1815995.

(12) Gollwitzer, C.; Bartczak, D.; Goenaga-Infante, H.; Kestens, V.; Krumrey, M.; Minelli, C.; Pálmai, M.; Ramaye, Y.; Roebben, G.; Sikora, A.; Varga, Z. A Comparison of Techniques for Size Measurement of Nanoparticles in Cell Culture Medium. Anal. Methods 2016, 8 (26), 5272-5282. https://doi.org/10.1039/C6AY00419A.

(13) Stetefeld, J.; McKenna, S. A.; Patel, T. R. Dynamic Light Scattering: A Practical Guide and Applications in Biomedical Sciences. Biophys. Rev. 201684 2016, 8 (4), 409-427. https://doi.org/10.1007/S12551-016-0218-6.

(14) Deborah K. Wilkins; Shaun B. Grimshaw; Véronique Receveur, +; Christopher M. Dobson; Jonathan A. Jones, § and; Smith*, L. J. Hydrodynamic Radii of Native and Denatured Proteins Measured by Pulse Field Gradient NMR Techniques $\dagger$.

Biochemistry 1999, 38 (50), 16424-16431. https://doi.org/10.1021/BI991765Q.

(15) Cabré, F.; Canela, E. I.; Canela, M. A. Accuracy and Precision in the Determination of Stokes Radii and Molecular Masses of Proteins by Gel Filtration Chromatography. $J$. Chromatogr. A 1989, 472 (C), 347-356. https://doi.org/10.1016/S00219673(00)94133-5.

(16) Franken, L. E.; Boekema, E. J.; Stuart, M. C. A. Transmission Electron Microscopy as a Tool for the Characterization of Soft Materials: Application and Interpretation. $A d v$. Sci. 2017, 4 (5), 1600476. https://doi.org/10.1002/ADVS.201600476.

(17) Schneider, M. C.; Telschow, R.; Mercier, G.; López-Martinez, M.; Scherzer, O.; Schütz, G. J. A Workflow for Sizing Oligomeric Biomolecules Based on Cryo Single Molecule Localization Microscopy. PLoS One 2021, 16 (1), e0245693. https://doi.org/10.1371/JOURNAL.PONE.0245693.

(18) Herling, T. W.; Levin, A.; Saar, K. L.; Dobson, C. M.; Knowles, T. P. J. Microfluidic Approaches for Probing Amyloid Assembly and Behaviour. Lab Chip 2018, 18 (7), 999-1016. https://doi.org/10.1039/c7lc01241a.

(19) Arter, W. E.; Levin, A.; Krainer, G.; Knowles, T. P. J. Microfluidic Approaches for the 
Analysis of Protein-Protein Interactions in Solution. Biophys. Rev. 2020, 1-11. https://doi.org/10.1007/s12551-020-00679-4.

(20) Moser, M. R.; Baker, C. A. Taylor Dispersion Analysis in Fused Silica Capillaries: A Tutorial Review. Anal. Methods 2021, 13 (21), 2357-2373.

https://doi.org/10.1039/D1AY00588J.

(21) Filipe, V.; Hawe, A.; Jiskoot, W. Critical Evaluation of Nanoparticle Tracking Analysis (NTA) by NanoSight for the Measurement of Nanoparticles and Protein Aggregates. Pharm. Res. 2010275 2010, 27 (5), 796-810. https://doi.org/10.1007/S11095-010-0073-2.

(22) Pal, N.; Verma, S. D.; Singh, M. K.; Sen, S. Fluorescence Correlation Spectroscopy: An Efficient Tool for Measuring Size, Size-Distribution and Polydispersity of Microemulsion Droplets in Solution. Anal. Chem. 2011, 83 (20), 7736-7744. https://doi.org/10.1021/AC2012637.

(23) Young, G.; Hundt, N.; Cole, D.; Fineberg, A.; Andrecka, J.; Tyler, A.; Olerinyova, A.; Ansari, A.; Marklund, E. G.; Collier, M. P.; Chandler, S. A.; Tkachenko, O.; Allen, J.; Crispin, M.; Billington, N.; Takagi, Y.; Sellers, J. R.; Eichmann, C.; Selenko, P.; Frey, L.; Riek, R.; Galpin, M. R.; Struwe, W. B.; Benesch, J. L. P.; Kukura, P. Quantitative Mass Imaging of Single Biological Macromolecules. Science (80-. ). 2018, 360 (6387), 423-427. https://doi.org/10.1126/SCIENCE.AAR5839.

(24) Mojarad, N.; Krishnan, M. Measuring the Size and Charge of Single Nanoscale Objects in Solution Using an Electrostatic Fluidic Trap. Nat. Nanotechnol. 201277 2012, 7 (7), 448-452. https://doi.org/10.1038/nnano.2012.99.

(25) Allen, S. M.; Balluffi, R. W.; Carter, W. C. Kinetics of Materials; J. Wiley \& Sons: Hoboken, NJ, USA, 2005.

(26) Vanderpoorten, O.; Babar, A. N.; Krainer, G.; Jacquat, R. P. B.; Challa, P. K.; Peter, Q.; Toprakcioglu, Z.; Xu, C. K.; Keyser, U. F.; Baumberg, J.; Kaminski, C. F.; Knowles, T. P. J. 2-Photon-Fabricated Nano-Fluidic Traps for Extended Detection of Single Macromolecules and Colloids in Solution. bioRxiv 2021, 2021.11.17.468989. https://doi.org/10.1101/2021.11.17.468989.

(27) Vanderpoorten, O.; Peter, Q.; Challa, P. K.; Keyser, U. F.; Baumberg, J.; Kaminski, C. F.; Knowles, T. P. J. Scalable Integration of Nano-, and Microfluidics with Hybrid Two-Photon Lithography. Microsystems Nanoeng. 201951 2019, 5 (1), 1-9. https://doi.org/10.1038/s41378-019-0080-3.

(28) Challa, P. K.; Kartanas, T.; Charmet, J.; Knowles, T. P. J. Microfluidic Devices Fabricated Using Fast Wafer-Scale LED-Lithography Patterning. Biomicrofluidics 2017, 11 (1), 014113. https://doi.org/10.1063/1.4976690.

(29) Duffy, D. C.; McDonald, J. C.; Schueller, O. J. A.; Whitesides, G. M. Rapid Prototyping of Microfluidic Systems in Poly(Dimethylsiloxane). Anal. Chem. 1998, 70 (23), 4974-4984. https://doi.org/10.1021/ac980656z.

(30) Cabrera, J. N.; Ruiz, M. M.; Fascio, M.; D’Accorso, N.; Minchev, R.; Dubois, P.; Lizarraga, L.; Negri, R. M. Increased Surface Roughness in Polydimethylsiloxane Films by Physical and Chemical Methods. Polym. 2017, Vol. 9, Page 331 2017, 9 (8), 331. https://doi.org/10.3390/POLYM9080331.

(31) Hoyer, W.; Antony, T.; Cherny, D.; Heim, G.; Jovin, T. M.; Subramaniam, V. Dependence of $\alpha$-Synuclein Aggregate Morphology on Solution Conditions. J. Mol. Biol. 2002, 322 (2), 383-393. https://doi.org/10.1016/S0022-2836(02)00775-1. 